\section{Achados polissonográficos em crianças portadoras de laringopatias}

\author{
Michele Themis Moraes Gonçalves ${ }^{1}$, Juliana \\ Sato $^{2}$, Melissa A. G. Avelino ${ }^{3}$, Gilberto U. Pizarro ${ }^{4}$, \\ Gustavo A. Moreira', Márcia Pradella Hallinan', \\ Reginaldo R. Fujita', Luc Louis Maurice Weckx ${ }^{8}$
}

Resumo

$\mathrm{O}$ exame diagnóstico padrão-ouro para SAOS na infância é a polissonografia. Quando pode ser efetuado, a polissonografia separa com clareza portadores de ronco primário de pacientes com apnéia (obstrutiva, central e mista) do sono. A causa mais freqüente da SAOS na infância é a hipertrofia adenoamigdaliana. Laringomalácia é a causa mais comum de estridor na infância, porém sua fisiopatologia permanece desconhecida. Entre as teorias mais aceitas estão a imaturidade do arcabouço cartilaginoso da laringe e/ou a imaturidade neuromuscular. Objetivo: Nossa proposta foi descrever os achados polissonográficos de crianças portadoras de laringomalácia e outras alterações laríngeas isoladas, ou seja, sem alterações nas vias aéreas superiores. Métodos: Foram selecionadas 29 crianças portadoras de alterações laríngeas exclusivas. Todas foram submetidas a exame otorrinolaringológico, nasofibrolaringoscopia e polissonografia. Foram tabulados dados relativos à idade, nasofibrolaringoscopia e polissonografia. Para análise, as crianças foram separadas em 2 grupos: portadores de laringomalácia e portadores de outras doenças laríngeas. Resultados: Dentre as 18 crianças com diagnóstico de laringomalácia, 18 apresentaram eventos respiratórios do tipo central, sendo a maioria dos episódios associados à dessaturação de oxigênio e alguns à bradicardia. Nesse mesmo grupo, 3 crianças apresentaram apnéia do tipo obstrutivo. Por outro lado, as 11 crianças portadoras de outras alterações laríngeas não apresentaram predominância entre um tipo ou outro de apnéia, 4 apresentaram eventos respiratórios do tipo central, 2 do tipo obstrutivo. Conclusão: A maioria dos pacientes acometidos por laringomalácia apresentou eventos respiratórios do tipo central quando avaliados pela polissonografia. Já os pacientes com outras doenças laríngeas não apresentaram predominância entre um tipo ou outro de apnéia.

\section{Polisomnographic findings on children with laryngopathies}

Palavras-chave: polissonografia, laringe, apnéia, crianças. Key words: Polysomnography, Larynx, Apnea, Children.

\section{Summary}

P - olysomnography is the goldstandard exam for child OSAS. When possible, polysomnography clearly distinguishes between those with isolated primary snoring and patients with sleep apnea (obstructive, central and mixed). The most common cause of OSAS in childhood is adenotonsillar hypertrophy. Laryngomalacia is the most common cause of stridor in childhood, though its physiopathology remains unknown. Among the most prominent theories are immaturity of the cartilaginous framework of the larynx and/or neuromuscular immaturity. Objective: Our proposal was to describe polysomnographic findings in children with laryngomalacia or other isolated laryngeal alterations, that is, without other alterations in the upper airways. Methods: The sample included 29 children with exclusively laryngeal alterations. All of them underwent an otorhinolaryngological exam, nasofibrolaryngoscopy and polysomnography. Information was recorded concerning age, nasofibrolaryngoscopy and polysomnography. For analysis, the children were divided into two groups: those with laryngomalacia and those with other laryngeal diseases. Results: Among the 18 children with a diagnosis of laryngomalacia, 18 had central breathing events, knowing that the majority had showed dessaturation of oxihemoglobin and bradicardia. In this same group, 3 children had obstrutives events. On the other hand, 11 children with other laryngeal alterations showed no predominance of one type or another of apnea. Of these, 4 had central type breathing events and 2 obstructive type. Conclusion: The majority of patients with laryngomalacia showed a central type apnea. Patients with various laryngeal diseases did not present a predominant type of apnea.

\footnotetext{
Trabalho premiado no IV Congresso Triológico de 2005

Médica, Especializanda em Otorrinolaringologia pela UNIFESP.

${ }^{2}$ Residente em Otorrinolaringologia pela UNIFESP-EPM, Médica.

${ }_{3}$ Pós-graduanda (Doutorado) do Departamento de Otorrinolaringologia da UNIFESP-EPM, Mestre em Otorrinolaringologia.

${ }^{4}$ Pós-graduando (mestrado) do Departamento de Otorrinolaringologia da UNIFESP-EPM, Otorrinolaringologista.

5 Mestre em pediatria pela UNIFESP-EPM, Professor da disciplina de Medicina e Biologia do Sono da UNIFESP-EPM.

${ }^{6}$ Mestre em Pediatria pela UNIFESP-EPM, Professora da Disciplina de Medicina e Biologia do Sono da UNIFESP-EPM.

7 Doutor em Otorrinolaringologia pela UNIFESP-EPM. Chefe de Clínica de Otorrinolaringologia Pediátrica da UNIFESP-EPM. UNIFESP-EPM.

${ }^{8}$ Doutor em Otorrinolaringologia pela UNIFESP-EPM. Chefe de Clínica de Otorrinolaringologia da UNIFESP-EPM.

Endereço para correspondência: Michele Themis Moraes Gonçalves Avenida Doutor Altino Arantes 870 apto. 43 Vila Clementino São Paulo SP 04042-004. Tel: (0xx11) 5089-6347 / 8167-0002 - E-mail: michelethemis@gmail.com

Este artigo foi submetido no SGP (Sistema de Gestão de Publicaç̃es) da RBORL em 31 de agosto de 2005. Artigo aceito em 13 de setembro de 2005.
} 


\section{INTRODUÇÃO}

Ronco e respiração oral são queixas muito comuns na infância, apresentando uma incidência que varia de 3 a 26\%. Na população pediátrica, verifica-se 1 a $3 \%$ de portadores da Síndrome da Apnéia Obstrutiva do Sono $(\mathrm{SAOS})^{1,2}$ sendo o ronco o sintoma mais comum.

Ronco é o nome dado ao som vibratório produzido na nasofaringe durante a inspiração. Chamamos de ronco primário, aquele não associado à apnéia, hipoventilação, hipoxemia, hipercapnia, sono agitado ou sonolência diurna excessiva.

A SAOS em crianças é caracterizada por episódios de obstrução parcial ou completa da via aérea superior que ocorrem durante o sono, geralmente acompanhados de queda na saturação de oxihemoglobina e/ou hipercapnia. A primeira série de casos de SAOS em crianças foi publicada em 1976 por Guilleminault et $\mathrm{al}^{3}$. A SAOS em crianças é diferente da SAOS em adultos. Em crianças, a doença abrange episódios de hipoventilação obstrutiva isolada, quadros de apnéia associada à hipoventilação obstrutiva, além de apnéia obstrutiva pura. Em outras palavras, as desordens respiratórias obstrutivas do sono são chamadas de SAOS mesmo quando não há episódios de apnéia.

Por definição, apnéia é a ausência de fluxo aéreo pela boca ou nariz. Pode ser causada por parada de movimentos respiratórios (apnéia do tipo central) ou por colabamento das vias aéreas superiores (apnéia do tipo obstrutiva). Quando a apnéia do tipo central e obstrutiva ocorrem simultaneamente, é chamada de mista. As apnéias do tipo obstrutiva e central em crianças, para serem consideradas relevantes, devem durar mais que 2 ciclos respiratórios e nem sempre estão associadas à hipoxemia ${ }^{4}$. Apnéia central de curta duração (menor que 15 segundos) é um achado comum durante o sono de crianças recémnascidas normais 5 .

Até o momento, não existe critério que defina um número aceitável de apnéias centrais. No entanto, se associadas à bradicardia ou cianose, independente de sua duração, é considerada significativa. Causas de origem cardíaca, hematológica, infecciosa, metabólica, neurológica, pulmonar, gastrointestinal e neuromuscular são citadas para a apnéia central, apesar de, na maioria das vezes, não ser identificada.

Entende-se por hipoventilação o decréscimo na ventilação pulmonar abaixo do mínimo necessário para manter a saturação de oxigênio normal. Assim como a apnéia, também pode ser de causa não-obstrutiva (menor estímulo respiratório central, anormalidades neuromusculares ou doenças restritivas pulmonares) ou obstrutiva (obstrução parcial das vias aéreas superiores levando a uma inadequada ventilação pulmonar).

Ao contrário dos adultos, nos quais o principal sin- toma de SAOS é sonolência diurna excessiva, as crianças são levadas ao médico, na maior parte das vezes, referindo ronco ou dificuldade respiratória durante o sono. Despertares noturnos, respiração bucal crônica, sonolência diurna excessiva, agitação psicomotora, movimentação excessiva noturna também são freqüentes. Crianças com SAOS podem apresentar baixo rendimento escolar, problemas disciplinares, distúrbios de atenção, alterações de humor (irritabilidade e agressividade), distúrbios de deglutição e alterações da voz ${ }^{30,31}$. Além disso, pode ocorrer déficit importante do crescimento, por alteração na secreção de GH durante o sono ${ }^{6-8}$. Outras hipóteses, porém ainda não comprovadas, são que o déficit pôndero-estatural seja secundário a maior gasto energético durante a respiração mais laboriosa ou devido a uma menor ingesta calórica causada por diminuição do apetite ou disfagia ${ }^{9-12}$. Formas graves podem estar associadas a cor pulmonale e óbito.

A fisiopatologia da SAOS em crianças não é totalmente conhecida. Sabe-se que não existe um mecanismo único envolvido e que, provavelmente, a SAOS em crianças com hipertrofia adenoamigdaliana seja diferente da SAOS em crianças com síndromes craniofaciais ou neurológicas.

O exame otorrinolaringológico é muito importante e começa pela inspeção da face, averiguando a existência de retrognatismo ou hipognatismo. A seguir, avalia-se o tamanho da língua e sua relação com a arcada dentária, a presença de hipertrofia amigdaliana, palato ogival e mordida aberta. Na cavidade nasal procura-se observar hipertrofia e/ou cianose de cornetos inferiores, desvios septais, presença de secreção, pólipos ou tumores. O exame com fibra óptica é necessário e muito importante, pois pode definir na criança os locais de estreitamento na via aérea superior, a relação da adenóide com a coana, da base da língua e orofaringe com a parede posterior da faringe, o colapso látero-lateral da amígdala, sinais de refluxo gastroesofágico ou alterações glóticas e subglóticas.

Como no adulto, o exame diagnóstico padrão-ouro para SAOS na infância é a polissonografia, para avaliar a gravidade e a conduta a ser assumida. Trata-se de um exame que necessita de profissionais habilidosos no trato com a criança, sendo muito importante a participação ativa dos pais. Quando pode ser efetuado, a polissonografia separa com clareza portadores de ronco primário de pacientes com apnéia (obstrutiva, central e mista) do sono.

Os critérios polissonográficos diagnósticos para SAOS em crianças foram definidos apenas recentemente. Em crianças, 1 ou mais episódios de apnéia do tipo obstrutivo por hora e/ou hipoventilação do tipo obstrutivo, caracterizado por pico de gás carbônico exalado (ETCO2) $\geq 53 \mathrm{mmHg}$, ETCO2 > $50 \mathrm{mmHg}$ em mais que 10\% do tempo total do sono ou ETCO2 > $45 \mathrm{mmHg}$ em mais que 60\% do tempo total do sono, são considerados anormais. IAH maior que 10 é considerado muito grave ${ }^{13}$. À polis- 
sonografia, roncadores primários apresentam índice de apnéia/hipopnéia (IAH) menor que 1 evento por hora, média da saturação de oxigênio durante o sono maior que $90 \%$ e pico de ETCO $2<53 \mathrm{mmHg}$ ou pico de ETCO2 $\geq$ $50 \mathrm{mmHg}$ em menos de $10 \%$ do tempo total do sono ${ }^{14}$. Em relação à avaliação da eficiência do sono, esta é considerada anormal quando menor ou igual a $85 \% 4$.

A causa mais freqüente da SAHOS na infância é a hipertrofia adenoamigdaliana ${ }^{6}$, tendo como fator agravante a rinite crônica hipertrófica. É muito importante averiguar também outras causas anatômicas (desvio de septo, polipose nasal, estenose coanal, macroglossia, micrognatia, laringomalácia, diafragma laríngeo), doenças congênitas e sindrômicas (Síndrome de Apert, Síndrome Cri du Chat, Síndrome de Crouzon, Síndrome de Down, Seqüência de Pierre Robin, Síndrome de Treacher-Collins, mucopolissacaridose, fibrose cística, Síndrome de ArnoldChiari, paralisia cerebral) entre outras (miopatia, doença neuromuscular, neoplasias, fatores familiares, refluxo gastroesofágico).

Laringomalácia é a causa mais comum de estridor na infância ${ }^{15}$, sendo sua prevalência desconhecida. Dados de literatura citam incidências que variam de 19,4 a 75\% $\%^{16,17}$. Tipicamente, aparece estridor após as primeiras semanas de vida que pode persistir até 18-24 meses. O estridor pode se intensificar na agitação, alimentação ou posição supina. Ele se deve a um colapso supraglótico secundário a excesso de mucosa da parede posterior de laringe, encurtamento de prega ariepiglótica ou desabamento de cartilagem epiglótica sobre a luz da laringe. A causa permanece em discussão. Há muitas teorias propostas para explicar essa fisiopatologia, desde desordens na formação da estrutura cartilaginosa da laringe e traquéia levando a uma maior flacidez das estruturas supraglóticas, alterações anatômicas e imaturidade neuromuscular ${ }^{18-21}$.

Em 1897, a teoria anatômica foi proposta por Sutherland \& Lack ${ }^{22}$ após um estudo de 18 casos de obstrução laríngea congênita, no qual concluiu-se que o distúrbio estava associado à imaturidade do tecido cartilaginoso da criança. Prescott ${ }^{23}$ complementou esta hipótese, estudando 40 pacientes com laringomalácia e notou que todos tinham pregas ariepiglótica curtas e 30\% apresentavam desordens neuromusculares.

Thompson e Turner ${ }^{24}$ demonstraram que prolapso medial passivo de supraglote poderia ser induzido a partir da denervação de laringes de crianças. Esse estudo, somado a outros como o de Perón et al. ${ }^{25}$ e Wiggs e Dinardo ${ }^{26}$, nos quais pacientes desenvolveram laringomalácia após sofrerem lesões neurológicas, afirmam a hipótese neurológica. A teoria neurológica explica a associação da laringomalácia com outras anormalidades neurológicas ${ }^{22,27}$. Belmont e Grundfast ${ }^{28}$ encontraram entre 30 crianças com laringomalácia estudadas 80\% de DRGE, 13\% de hipotonia e $10 \%$ de apnéia central.
O diagnóstico da laringomalácia é feito através da laringoscopia flexível durante a respiração espontânea. Geralmente apresenta resolução espontânea. Relatos da literatura indicam que é pouco freqüente a laringomalácia ser severa a ponto de resultar em cianose, dificuldade de alimentação, cor pulmonale, apnéia ou falência de crescimento. Quando acontece, tradicionalmente, pacientes com laringomalácia severa são tratados com traqueostomia, mas há inúmeros outros tratamentos cirúrgicos (ressecção da prega ariepiglótica, epiglotoplastia e supragloteplastia).

\section{OBJETIVO}

Nossa proposta foi descrever os achados polissonográficos de crianças portadoras de laringomalácia e outras alterações laríngeas isoladas, ou seja, sem alterações nas vias aéreas superiores.

\section{MATERIAIS E MÉTODOS}

O trabalho foi realizado no Centro do Respirador Oral da Disciplina de Otorrinolaringologia Pediátrica da Universidade Federal de São Paulo e no Instituto do Sono no Departamento de Psicobiologia da Universidade Federal de São Paulo. Durante o período de 1999 a 2004 foram selecionadas 29 (vinte e nove) crianças portadoras de laringomalácia ou outras alterações laríngeas. A idade variou de 11 dias de vida a 8 anos e 10 meses. Todas foram submetidas a exame otorrinolaringológico, nasofibrolaringoscopia e polissonografia. Foram incluídos somente pacientes com queixas de ronco e estridor laríngeo ou alteração vocal, que ao exame fibroscópico apresentavam laringomalácia ou outra alteração laríngea isoladamente. Foi considerado como laringomalácia a presença de excesso de mucosa em parede posterior de laringe, encurtamento de prega ariepiglótica ou desabamento de cartilagem epiglótica sobre luz laríngea.

Foram excluídas as crianças portadoras de hipertrofia adenoamigdaliana, malformações craniofaciais ou síndromes genéticas.

Os pacientes foram submetidos à polissonografia diurna no Instituto do Sono da UNIFESP-EPM, onde o exame foi realizado com o paciente dormindo em cama confortável, num quarto escuro e silencioso. Os parâmetros eletrofisiológicos e cardio-respiratórios foram registrados em sistema computadorizado (Alice ${ }^{\circledR}$ ): eletroencefalograma (4 canais), eletromiograma submentoniano e tibial, eletrooculograma direito e esquerdo, fluxo de ar oronasal, movimento de tórax e abdômen, microfone, saturação da oxihemoglobina ( $\mathrm{SaO} 2)$, e posição no leito. O paciente foi estudado em ar ambiente. Foram tabulados dados relativos à idade, nasofibrolaringoscopia e polissonografia. Para análise, as crianças foram separadas em 2 grupos: portadores de laringomalácia e portadores de outras doenças laríngeas (estenose subglótica, hemangioma, sinéquia, 
paralisia de prega vocal, nódulo vocal, fenda vocal, fixação de aritenóide e/ou espessamento de prega vocal).

Para análise estatística dos dados, foi empregado o teste de Fisher, que não mostrou significância entre os resultados.

Todos os pacientes apresentam termo de ciência e termo de consentimento informado, e este trabalho foi aprovado pelo comitê de ética. (Protocolo nº 0122/02).

\section{RESULTADOS}

Os resultados encontrados nas polissonografias realizadas entre os pacientes portadores de laringomalácia e outras lesões laríngeas estão representados, respectivamente, nas Tabelas 1 e 2 .

Dentre as 18 crianças com diagnóstico de laringomalácia, 18 apresentaram eventos respiratórios do tipo central, sendo a maioria dos episódios associados à dessaturação de oxigênio e alguns à bradicardia. Nesse mesmo grupo, 3 crianças apresentaram apnéia do tipo obstrutivo. Em relação às 11 crianças portadoras de outras alterações laríngeas, 4 apresentaram eventos respiratórios do tipo central, 2 do tipo obstrutivo.

A saturação de oxigênio durante do sono das crianças estudadas variou de 76 a 98\%.

O paciente INJ, de 3 meses de idade, é portador de laringomalácia considerada grave, pois apresentava saturação de oxigênio basal (acordada e em repouso) de $89 \%$. Apesar de o paciente ter apresentado 2 eventos respiratórios do tipo central, houve uma melhora na saturação de oxigênio durante o sono.

\section{DISCUSSÃO}

De 3 a 26\% da população infantil procura o consultório do especialista com queixas de ronco e respiração oral, sendo que apenas 1 a 3\% apresenta a Síndrome da Apnéia Obstrutiva do Sono (SAOS). A doença é suspeitada através de dados da anamnese, porém esta é insuficiente para separar os roncadores primários dos portadores de SAOS. Neste caso, está indicada a realização de polissonografia que, além de definir o diagnóstico, caracteriza o tipo de evento respiratório ocorrido, ou seja, se obstrutivo, central ou misto, além de avaliar a gravidade da doença, através do Índice de Apnéia e Hipopnéia (IAH) e da saturação de oxigênio. É importante relembrar que, em relação à fisiopatologia e critérios diagnósticos polissonográficos, a SAOS em crianças é considerada uma entidade diferente da do adulto.

O exame otorrinolaringológico, associado à nasofibrolaringoscopia, identifica a maior parte das causas da apnéia. Apesar de na maioria das vezes ser causada por hipertrofia adenoamigdaliana, não se pode deixar de averiguar outras causas, entre elas as doenças laríngeas.

Tabela 1. Dados de polissonografia observados nos pacientes portadores de laringomalácia.

\begin{tabular}{lccccccccccccc}
\hline Paciente & Idade & naso & sat vig & SC & SA & NED & NEC & IAC & NEO & nadir & FC V & FC A & FC Q \\
\hline TMS & $6 \mathrm{~m}$ & LM & $98 \%$ & $96 \%$ & $93 \%$ & 1 & 2 & 0.9 & 0 & $89 \%$ & 167,5 & 135,1 & 135,6 \\
LAB & 5m & LM & $95 \%$ & $92 \%$ & $92 \%$ & 1 & 1 & 0.4 & 0 & $89 \%$ & 145,0 & 123,5 & 126,0 \\
ECM & 5m & LM & $90 \%$ & $92 \%$ & $93 \%$ & 1 & 1 & 0.7 & 0 & $85 \%$ & 173,0 & 151,4 & 158,8 \\
GSS & 6m & LM & $96 \%$ & $96 \%$ & $96 \%$ & 20 & 20 & 7.7 & 0 & $88 \%$ & 163,2 & 139,1 & 134,2 \\
DASS & 9m & LM & $98 \%$ & $97 \%$ & $98 \%$ & 7 & 7 & 1.0 & 0 & $92 \%$ & 138,2 & 126,9 & 119,8 \\
SNR & 5m & LM & $95 \%$ & $95 \%$ & $95 \%$ & - & 1 & 0.5 & 0 & $95 \%$ & indisp & indisp & indisp \\
TNB & 2m & LM & $96 \%$ & $94 \%$ & $96 \%$ & 7 & 7 & 2.6 & 1 & $90 \%$ & 174,9 & 136,9 & 141,0 \\
SMS & $11 \mathrm{~m}$ & LM & $92 \%$ & $90 \%$ & $89 \%$ & 3 & 12 & 7.5 & 0 & $76 \%$ & 158,7 & 141,9 & 138,4 \\
PJS & 3m & LM & $98 \%$ & $96 \%$ & $95 \%$ & 3 & 5 & 2.0 & 0 & $91 \%$ & 160,2 & 130,1 & 134,0 \\
MGF & 6m & LM & $97 \%$ & $95 \%$ & $93 \%$ & 5 & 6 & 2.8 & 0 & $87 \%$ & indisp & indisp & indisp \\
LFSS & $21 \mathrm{~d}$ & LM & $96 \%$ & $96 \%$ & $92 \%$ & 6 & 8 & 4.2 & 0 & $91 \%$ & 164,3 & 151,7 & 138,4 \\
KMS & 7m & LM & $97 \%$ & $97 \%$ & $97 \%$ & 10 & 12 & 4.6 & 0 & $88 \%$ & 177,9 & 151,8 & 152,1 \\
KSS & $3 \mathrm{~m}$ & LM & $96 \%$ & $96 \%$ & $95 \%$ & 4 & 18 & 6.9 & 0 & $92 \%$ & 154,6 & 137,8 & 134,0 \\
ENR & 2m & LM & $99 \%$ & $98 \%$ & $98 \%$ & 3 & 18 & 8.9 & 0 & $93 \%$ & indisp & indisp & indisp \\
INJ & 3m & LM & $89 \%$ & $93 \%$ & $93 \%$ & - & 2 & 1.0 & 0 & $90 \%$ & 182,4 & 143,6 & indisp \\
EGS & $17 \mathrm{~m}$ & LM & $98 \%$ & $95 \%$ & $96 \%$ & - & 24 & 2.6 & 7 & $92 \%$ & 129,4 & 129,0 & 124,3 \\
BNM & 4m & LM & $96 \%$ & $96 \%$ & $96 \%$ & 1 & 2 & 1.5 & 0 & $92 \%$ & 152,5 & 121,8 & 119,1 \\
ASO & 4m & LM & $96 \%$ & $94 \%$ & $94 \%$ & 13 & 30 & 25.0 & 1 & $86 \%$ & 136,8 & 125,0 & 122,4 \\
\hline
\end{tabular}


Tabela 2. Dados de polissonografia observados nos pacientes portadores de outras doenças laríngeas.

\begin{tabular}{|c|c|c|c|c|c|c|c|c|c|c|}
\hline Paciente & Idade & naso & sat vig & SC & SA & NED & NEC & IAC & $\mathrm{IAHO}$ & nadir \\
\hline CRM & $6 a$ & nódulos vocais bilateral & $99 \%$ & $98 \%$ & $98 \%$ & indisp & 0 & 0.4 & & $92 \%$ \\
\hline JCS & $2 a 8 m$ & mucosa reg post desab glote & $99 \%$ & $99 \%$ & $99 \%$ & indisp & 0 & indisp & & $89 \%$ \\
\hline RAS & $7 a$ & espessamento ppvv bilt* & $98 \%$ & $98 \%$ & $98 \%$ & indisp & 3 & indisp & & $94 \%$ \\
\hline SFC & $7 a$ & nódulos vocais bilateral & $98 \%$ & $96 \%$ & $96 \%$ & indisp & 0 & indisp & 0.9 & $89 \%$ \\
\hline MHS & $1 a$ & $\begin{array}{c}\text { estenose subglótica ant } \\
\text { obstr } 70 \%\end{array}$ & $96 \%$ & $96 \%$ & $97 \%$ & indisp & 2 & indisp & & $94 \%$ \\
\hline OGST & $1 a$ & $\begin{array}{l}\text { encurtamento de prega arie- } \\
\text { piglótica }\end{array}$ & $93 \%$ & $92 \%$ & $92 \%$ & indisp & 16 & indisp & & $87 \%$ \\
\hline AAA & $6 a$ & papilomatose obstr laringe & $98 \%$ & $96 \%$ & $95 \%$ & indisp & 0 & indisp & & $92 \%$ \\
\hline
\end{tabular}

\section{Legenda:}

naso: diagnóstico à nasofibrolaringoscopia IAC: Índice de Apnéia Central

Sat vig: saturação na vigília NEO: número de eventos obstrutivos

SC: sono calmo nadir: menor saturação da oxi-hemoglobina

SA: sono ativo FC V: freqüência cardíaca na vigília

NED: número de eventos de dessaturação FC A: freqüência cardíaca no sono ativo

NEC: número de eventos centrais FC Q: freqüência cardíaca no sono calmo

indisp: indisponível

A fisiopatologia da laringomalácia ainda permanece controversa. Entre as teorias mais aceitas, tem-se a imaturidade do sistema nervoso. Achado concordante é observado no trabalho de Milczuk \& Johnson ${ }^{(29)}$, no qual crianças com laringomalácia apresentavam como comorbidades DRGE, prematuridade e atraso de DNPM entre outras, todas relacionadas a algum grau de imaturidade neurológica.

Apnéia central de curta duração é um achado considerado normal durante o sono de recém-nascidos e, até o momento, não existe um critério que defina um número aceitável de apnéias centrais. Este tipo de apnéia, para ser considerado relevante, deve durar mais que 2 ciclos respiratórios ou estar associado à bradicardia e/ou cianose. Dentre as 18 crianças com diagnóstico de laringomalácia, 18 apresentaram eventos respiratórios do tipo central, sendo a maioria dos episódios associados a dessaturação de oxigênio e alguns a bradicardia. Nesse mesmo grupo, 3 crianças apresentaram apnéia do tipo obstrutivo. Em relação às 11 crianças portadoras de outras alterações laríngeas, 4 apresentaram eventos respiratórios do tipo central, 2 do tipo obstrutivo.

O número de eventos em geral mostrou relação com a gravidade da dessaturação. Porém dois casos fogem à maioria: podemos citar o caso de ENR que, apesar de ter apresentado 18 eventos respiratórios, nenhum deles foi associado à dessaturação de oxigênio abaixo de 93\%, enquanto OGST apresentou praticamente o mesmo número de eventos, porém com dessaturação de oxigênio importante (87\%).

\section{CONCLUSÃO}

A maioria das crianças portadoras de laringomalácia ou outras doenças laríngeas não apresentou alterações respiratórias significativas durante o sono. Mas o grupo de lactentes portadores de laringomalácia apresentou mais apnéias do tipo central. Nesse grupo, embora a maioria dos eventos respiratórios possam ser considerados como benignos, sua maior freqüência sugere a presença de obstrução parcial das vias aéreas superiores.

Visto o achado de nosso trabalho, acreditamos que a avaliação dos distúrbios respiratórios do sono em pacientes portadores de doenças laríngeas, utilizando-se de métodos mais modernos providos de sensor de pressão para detecção do fluxo aéreo nasal, possa trazer novos dados para o entendimento da fisiopatologia dessas doenças e é isto que nos propomos a fazer como continuação deste trabalho. 


\section{REFERÊNCIAS BIBLIOGRÁFICAS}

1. Ali NJ, Pitson DJ, Stradling JR. Snoring, sleep disturbance and behaviour in 4-5 year olds. Arch Dis Child 1993;68:360-6.

2. Gislason T, Benediktsdottir B. Snoring, apneic episodes, and nocturnal hypoxemia among children 6 months to 6 years old. Chest 1995;107:963-6.

3. Guilleminault C, Eldrige FL, Simmons FB, Dement WC. Sleep apnea in eight children. Pediatrics 1976;58(1):23-30.

4. Carroll JL, Loughlin GM. In Ferber R, Kryger M. Principles and Practice of sleep medicine in the child. WB Saunders Company; 1995:163-91.

5. Guilleminault C. In: Guilleminault C. Sleep and its desorders in children. New York: Raven Press; 1987:195.

6. Bar A, Tarasiuk A, Segev Y, Phillip M, Tal A. The effect of adenotonsillectomy on serum insulin-like growth factor-I and growth in children with obstructive sleep apnea syndrome. J Pediatr 1999;135(1):7680.

7. Grunstein RR, Handelsman DJ, Lawrence SJ, Blackwell C, Caterson ID, Sullivan CE. Neuroendocrine dysfunction in sleep apnoea: reversal by continuous positive airways pressure therapy. J Clin Endocrinol Metab 1989;68:352-8.

8. Nieminen P, Lopponen T, Tolonen U, Lanning P, Knip M, Lopponen H. Growth and biochemical markers of growth in children with snoring and obstructive sleep apnea. Pediatrics 2002;109(4):55.

9. Williams EF, Woo P, Miller R, Kellman RM. The effects of adenotonsillectomy on growth in young children. Otolaryngol Head Neck Surg 1991;104:509-16.

10. Ahlqvist-Rastad J, Hultcrantz E, Melander H, Svanhom H. Body growth in relation to tonsillar enlargement and tonsillectomy. Int J Pediatr Otorhinolaryngol 1992;24:55-61.

11. Stradling JR, Thomas G, Warley HRA, Williams P, Freeland A. Effect of adenotonsillectomy on nocturnal hypoxaemia, sleep disturbance, and symptoms in snoring children. Lancet 1990;335:249-53.

12. Marcus CL, Carroll JL, Koerner CB, Hamer A, Lutz J, Louglin GM. Determinants of growth in children with obstructive sleep apnea syndrome. J Pediatr 1994;125:556-62.

13. Marcus CL, Omlin KJ, Basinki DJ, Bailey SL, Rachal AB, Von Pechmann WS, Keens TG, Ward SL. Normal polysomnographic values for children and adolescents. Am Rev Respir Dis 1992;146 (5 Pt1):1235-9.

14. Marcus CL, Hamer A, Loughlin GM. Natural history of primary snoring in children. Pediat Pulmonol 1998;26 (1):6-11.
15. Richardson MA, Cotton RT. Anatomic abnormalities of pediatric airway. Pediatr Clin North Am 1984;31(4):821-34.

16. Rupa V, Raman R. Aetiological profile of paediatric laryngeal stridor in an Indian hospital. Ann Trop Paediatr 1991;11(2):137-41.

17. Lane RW, Wieder DJ, Steinem C, Marin-Padilla M. Laryngomalacia:a review and case report of surgical treatment with resolution of pectus excavatum. Arch Otolatyngol 1984;110(8):546-51.

18. Zalzal GH, Anon JB, Cotton RT. Epiglottoplasty for the treatment of laryngomalacia. Ann Otol Rhinol Laryngol 1987;96:72-6.

19. Belmont JR, Grundfast K:Congenital laryngeal stridor (laryngomalacia):Etiologic factors and associated disorders. Ann Otol Rhinol Laryngol 1984;93:430-7.

20. Schulman JB, Hollister DW, Thibeault DW, Krugman ME. Familial laryngomalacia: a case report. Laryngoscope 1976;86(1):84-91.

21. McSwiney PF, Cavanagh NP, Langoth P. Outcome in congenital stridor (laryngomalacia). Arch Dis Child 1977;52:215-8.

22. Sutherland GA, Lack HL. Congenital laryngeal obstruction. Lancet 1897;2:653-5.

23. Prescott CA. The current status of corrective surgery for laryngomalacia. Am J Otolaryngol 1991;12(4):230-5.

24. Thompson J, Turner A. On the causation of congenital laryngeal stridor of infants. Br Md J 1900;2:1561-3.

25. Peron DL, Graffino DB, Zenker DO. The redundant aryepiglotic fold:report of a new cause of stridor. Laryngoscope 1988;98(6 Pt1):659-63.

26. Wiggs WJ, DiNardo LJ. Acquired laryngomalacia:resolution after neurologic recovery. Otolaryngol Head Neck Surg 1995;112(6):773-6.

27. Apley J. The infant with stridor: a follow-up survey of 80 cases. Arch Dis Child 1953;28:423-35.

28. Belmont JR, Grundfast K. Congenital laryngeal stridor (laryngomalacia):etiologic factors and associated disorders. Ann Otol Rhinol Laryngol 1984;93(5 Pt 1):430-7.

29. Milczuk HA, Johnson SM. Effect on families and caregivers of caring for a child with laryngomalacia. Ann Otol Rhinol Laryngol 2000;109(4):348-54

30. Kaemingk KL, Pasvogel AE, Goodwin JL, Mulvaney SA, Martinez F, Enright PL, Rosen GM, Morgan WJ, Fregosi RF, Quan SF. Learning in children and sleep disordered breathing:findings of the Tucson Children's Assessment of Sleep Apnea (tuCASA) prospective cohort study. J Int Neuropsychol Soc 2003;9(7):1016-26.

31. Archbold KH, Giordani B, Ruzicka DL, Chervin RD. Cognitive executive dysfunction in children with mild sleep-disordered breathing. Biol Res Nurs 2004;5(3):168-76. 